\title{
Peer Reviewers and Book Reviewers for 2012
}

The editors are extremely grateful for the contributions of the many peer reviewers and book reviewers who provided excellent, detailed, and constructive reviews of materials received by Physiotherapy Canada. Without their ongoing help and support, it would be impossible to provide the calibre of articles and book reviews that were published in 2012.

\section{PEER REVIEWERS FOR RECEIVED ARTICLES}

Agnidis, Zoe

Alcock, Greg

Anderson, Cathy

Bailey, Christine

Balogh, Rob

Barclay-Goddard, Ruth

Boe, Shaun

Budd, Lois

Busch, Angela

Cauraugh, James

Cheifetz, Oren

Chesworth, Bert

Coman, Liliana

Connelly, Denise

Culham, Elsie

Davis, Aileen

Debigare, Richard

Dechman, Gail

Delahunt, Eamonn

Deusingers, Susan

Dowthwaire, Jodi

Dubayova, Tatiana

Eason, Jane

El-Ansary, Doa

Ellerton, Cindy
Everett, Bethan

Forhan, Mary

Forwell, Lorie

Frank, Andrew

Gabel, Philip

Galea, Victoria

Giangregorio, Lora

Harris, Susan

Hoens, Alison M.

Holzman Weppler, Cynthia

Hopkins-Rosseel, Diana

Howe, Jo-Anne

Hoyles, Cathy

Jaglal, Susan

Keus, Samyra

King, Judy

Landry, Michel D.

Lonnroos, Eija

Macdonald, Heather

MacPhail, Ann

Manske, Sarah

McEwen, Sara

McFadyen, Brad

Miller, Pat

Morris, David
Nayer, Marla

O'Brien, Kelly

O'Sullivan, Kieran

Patman, Shane

Phadke, Chetan

Ploughman, Michelle

Pollock, Courtney

Pool, Jan

Rauch, Frank

Rennie, Sandy

Rocabado Seaton, Mariano

Salbach, Nancy M.

Sirtori, Valeria

Sran, Meena

Stokes, Moya

Stratford, Paul

Streiner, David

Swinamer, Jenneth

Thompson, Marlene

Uusi-Rasi, Kirsti

Von Piekartz, Harry

Walton, David

Webber, Sandra

Weinberg, Leah

Wessel, Jean

\section{BOOK REVIEWERS FOR PUBLISHED REVIEWS}

Andreoli, Angie

Cleaver, Shaun

Davies, Robyn
Ellerton, Cindy

Garrett, Jeff

Moody, Kim
Smith, Meredith

Waugh, Esther

\section{COCHRANE COLLABORATION}

The editors also thank Ruth Barclay-Goddard and Ted Stevenson for contributing Cochrane reviews in 2012. 